\title{
Comunicação
}

[Communication $]$

\section{Casuística dos exames de diagnóstico por imagem na medicina de animais selvagens - 2009 a 2010}

[Casuistics of diagnostic imaging in wildlife medicine - from 2009 to 2010]

\author{
Z. Bortolini, P.M. Matayoshi, R.V. Santos, D.P. Doiche, V.M.V. Machado, \\ C.R. Teixeira, L.C. Vulcano
}

Universidade Estadual Paulista -Botucatu, SP

\begin{abstract}
Diversas espécies de animais selvagens, com inúmeras particularidades fisiológicas são conhecidas atualmente, dificultando a realização de um exame clínico eficiente (Augusto, 2007). Entretanto, é crescente o número de estudos com esses animais, o que tem contribuído para um melhor conhecimento da anatomia e fisiologia das espécies, auxiliando, assim, os clínicos e cirurgiões quanto ao diagnóstico definitivo, ao prognóstico e à definição da melhor terapia (Mackey et al., 2008). Nesse processo de desenvolvimento, as técnicas de diagnóstico por imagem, como radiografia, ultrassonografia, tomografia computadorizada e ressonância magnética, têm sido utilizadas na complementação do exame clínico (Valente, 2007).
\end{abstract}

$\mathrm{O}$ serviço de diagnóstico por imagem se expandiu rapidamente na medicina de animais selvagens devido à combinação de tecnologias avançadas, como equipamentos portáteis, ao aprimoramento de técnicas anestésicas, ao condicionamento comportamental e ao maior conhecimento da fisiologia desses animais (Suedmeyer, 2006).

O exame radiográfico, indubitavelmente, é uma importante ferramenta para auxiliar o médico veterinário no diagnóstico e na pesquisa de várias enfermidades, visto que sua relação custo-benefício o torna a primeira escolha como método complementar de diagnóstico por imagem (Pinto, 2007). Porém, em algumas classes taxonômicas, como a dos répteis, que não possuem gordura mesentérica, a interpretação e a visibilização de órgãos da cavidade celomática no exame radiográfico ficam limitadas, uma vez que, devido à redução do contraste entre os tecidos, a

Recebido em 6 de março de 2012

Aceito em 12 de março de 2013

E-mail: zarabortolini@yahoo.com.br cavidade é visibilizada como uma estrutura única (Mitchell, 2009).

Outro método diagnóstico não invasivo que tem demonstrado resultados excelentes é a ultrassonografia. Suas aplicações incluem monitoramento da função reprodutiva e diagnóstico de afecções por meio da análise das mudanças anatômicas e topográficas dos órgãos. Além disso, auxilia em outras técnicas diagnósticas, como a biópsia guiada por ultrassom (Sainsbury e Gili, 1991; Stetter, 2006). A ultrassonografia e a radiografia são modalidades de imagem que fazem parte da rotina clínica, enquanto novas técnicas, como a tomografia computadorizada (TC), a ressonância magnética (RM) e a cintilografia nuclear, são utilizadas com menor frequência (Mackey et al., 2008) em razão do alto custo e da disponibilidade dos aparelhos. Atualmente, a TC está cada vez mais difundida e utilizada na rotina como uma técnica não invasiva e de curta duração (Gumpenberger e Henninger, 2001; Mackey et al., 2008), devido à maior acessibilidade e à diminuição do custo do exame.

Realizou-se um estudo retrospectivo dos prontuários dos animais selvagens encaminhados ao Setor de Diagnóstico por Imagem da Faculdade de Medicina Veterinária e Zootecnia (FMVZ) da Universidade Estadual Paulista (Unesp) - Campus de Botucatu, para compilação de dados, os quais foram analisados e agrupados conforme a modalidade de imagem (radiologia, ultrassonografia e tomografia computadorizada) e classe taxonômica (aves, répteis e mamíferos). Os procedimentos de diagnóstico por imagem foram divididos de acordo com $\mathrm{o}$ sistema acometido: tegumento comum, sistema cardiorrespiratório, osteomuscular (esqueleto 
apendicular) e neurológico (esqueleto axial). Nos mamíferos, foram diferenciadas a cavidade abdominal e a torácica, enquanto nas aves e répteis considerou-se apenas a cavidade celomática. Os exames que não apresentaram alterações radiográficas foram classificados como "nada digno de nota" (NDN).

Durante o período de janeiro de 2009 a dezembro de 2010, foi encaminhado ao Setor de Diagnóstico por Imagem da FMVZ - Unesp - Botucatu um tota de 191 animais selvagens, sendo 45\% (86/191) aves, 38\% (73/191) mamíferos e 17\% (32/191) répteis.

Foram realizados 322 exames imaginológicos, sendo 291 exames radiográficos, dos quais 99 foram exames de acompanhamento terapêutico (34\%); 20 exames tomográficos; e 11 exames ultrassonográficos. Em alguns animais foi realizado mais de um método de diagnóstico por imagem, e os dados obtidos distribuídos de acordo com a classe taxonômica (Fig. 1).

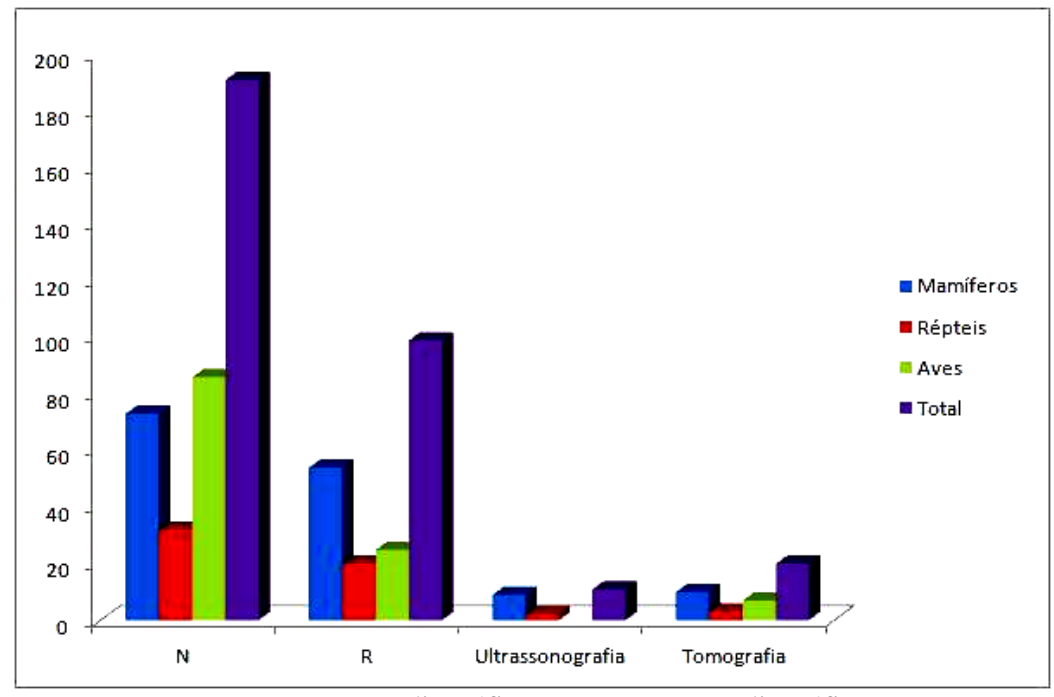

$\mathrm{N}=$ casos novos radiográficos, $\mathrm{R}=$ retornos radiográficos.

Figura 1. Distribuição dos atendimentos do Serviço de Diagnóstico por Imagem da FMVZ - Unesp - Botucatu, segundo a espécie e a modalidade de diagnóstico por imagem realizada, no período de janeiro 2009 a dezembro 2010.

Dos 191 novos exames radiográficos foram obtidos 203 diagnósticos, uma vez que alguns exames propiciaram o diagnóstico de mais de uma alteração. Destes diagnósticos, 92/203 (45\%) foram em aves, seguidos por 75/203 em mamíferos $(37 \%)$ e 36/203 em répteis (18\%). Quanto aos retornos, a maior incidência de acompanhamentos radiográficos foi de mamíferos $(55 \%)$, seguidos pelas aves $(25 \%)$ e répteis $(20 \%)$. Não foram necessárias reavaliações nos casos de exames ultrassonográficos e tomográficos no presente estudo.

Do número total de controles radiográficos osteomusculares (73), 60 foram acompanhamentos de lesões pós-traumáticas, sendo que a maior porcentagem foi realizada em mamíferos $65 \%$ $(39 / 60)$, seguida por $25 \%(15 / 60)$ em aves e $10 \%$ (6/60) em répteis (Tab. 1).

Tabela 1. Número de diagnósticos radiográficos distribuídos de acordo com a região de acometimento em cada classe de animais

\begin{tabular}{|c|c|c|c|c|c|c|c|c|c|c|c|c|c|c|}
\hline \multirow{2}{*}{ Classe } & \multicolumn{2}{|c|}{ TGC } & \multicolumn{2}{|c|}{ SN } & \multicolumn{2}{|c|}{$\mathrm{OM}$} & \multicolumn{2}{|c|}{$\mathrm{CC}$} & \multicolumn{2}{|c|}{ CT } & \multicolumn{2}{|c|}{$\mathrm{CA}$} & \multicolumn{2}{|c|}{ NDN } \\
\hline & $\mathrm{N}$ & $\mathrm{R}$ & $\mathrm{N}$ & $\mathrm{R}$ & $\mathrm{N}$ & $\mathrm{R}$ & $\mathrm{N}$ & $\mathrm{R}$ & $\mathrm{N}$ & $\mathrm{R}$ & $\mathrm{N}$ & $\mathrm{R}$ & $\bar{N}$ & $\mathrm{R}$ \\
\hline Mamíferos & - & 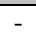 & 8 & 3 & 43 & 48 & - & - & 2 & - & 8 & 2 & 14 & 1 \\
\hline Répteis & - & - & 3 & - & 16 & 9 & 13 & 11 & - & - & - & - & 4 & - \\
\hline Aves & 5 & 3 & 2 & - & 53 & 16 & 20 & 5 & - & - & - & - & 12 & 1 \\
\hline
\end{tabular}

$\mathrm{N}=$ casos novos, $\mathrm{R}=$ retornos, $\mathrm{TGC}=$ tegumento comum, $\mathrm{SN}=$ sistema nervoso, $\mathrm{OM}=$ osteomuscular, $\mathrm{CC}=$ cavidade celomática, $\mathrm{CT}=$ cavidade torácica, $\mathrm{CA}=$ cavidade abdominal, $\mathrm{NDN}=$ nada digno de nota. 


\section{Casuística dos exames...}

Com relação à área de acometimento, a maior incidência de alterações foi observada no sistema osteomuscular, no qual 185 exames foram realizados, sendo 73 reavaliações e 112 novos diagnósticos. As aves representaram a maior casuística de lesões osteomusculares (53/112), seguidas de mamíferos (43/112) e répteis $(16 / 112)$ (Tab. 2). A maioria das lesões osteomusculares foram decorrentes de traumas $(89 \%)$.

Tabela 2. Exames radiográficos realizados dentro de cada classe por sistemas acometidos

\begin{tabular}{|c|c|c|c|c|c|c|c|}
\hline \multirow{2}{*}{\multicolumn{2}{|c|}{ Sistema/Classe }} & \multicolumn{2}{|c|}{ Aves } & \multicolumn{2}{|c|}{ Répteis } & \multicolumn{2}{|c|}{ Mamíferos } \\
\hline & & $\mathrm{N}$ & $\mathrm{R}$ & $\mathrm{N}$ & $\mathrm{R}$ & $\mathrm{N}$ & $\mathrm{R}$ \\
\hline & Traumático & 49 & 15 & 13 & 6 & 38 & 39 \\
\hline \multirow{2}{*}{ Osteoarticular } & Metabólico & 2 & - & 3 & 3 & 3 & 9 \\
\hline & Infeccioso & 2 & 1 & - & - & 2 & - \\
\hline \multicolumn{2}{|c|}{ Sistema nervoso } & 2 & - & 3 & - & 8 & 3 \\
\hline \multicolumn{2}{|c|}{ Cardiorrespiratório } & 11 & 1 & 7 & 2 & 2 & - \\
\hline \multicolumn{2}{|c|}{ Digestório } & 9 & 4 & 4 & 2 & 2 & 2 \\
\hline \multicolumn{2}{|c|}{ Tegumento comum } & 5 & 3 & - & - & - & - \\
\hline \multirow{2}{*}{\multicolumn{2}{|c|}{ Genitourinário }} & - & - & 2 & 7 & 6 & - \\
\hline & NDN & 12 & 1 & 4 & - & 14 & 1 \\
\hline
\end{tabular}

$\mathrm{N}=$ casos novos, $\mathrm{R}=$ retornos.

Já as áreas menos acometidas radiograficamente nos novos casos foram: cavidade torácica com $1 \%$ (2/203), tegumento comum com 3\% (5/203), cavidade abdominal com 4\% (08/203), sistema nervoso com $6 \%$ (13/203), cavidade celomática de aves com 6\% (13/203) e de répteis com $10 \%$ (20/203). Os casos em que não foram visibilizadas alterações radiográficas (NDN) representaram $15 \%$ (30/203) (Tab. 2).
Foram realizados 20 exames tomográficos, resultando em 21 diagnósticos, sendo que $45 \%$ destes decorreram de lesões no sistema nervoso $(10 / 21), 27 \%$ no osteomuscular (3/21), 27\% no genitourinário $(3 / 21), 9 \%$ no digestório $(2 / 21)$ e $14 \%$ no cardiorrespiratório (3/21), como discriminado na Tab. 3. Apenas um exame não apresentou um diagnóstico conclusivo.

Tabela 3. Número de exames tomográficos realizados dentro de cada classe taxômica e distribuídos de acordo com os sistemas

\begin{tabular}{llccc}
\multicolumn{2}{c}{ Sistema/ Classe } & Aves & Répteis & Mamíferos \\
\hline \multirow{2}{*}{ Osteomuscular } & Traumático & 1 & - & - \\
& Metabólico & - & 1 & - \\
Sistema nervoso & Infeccioso & - & - & 1 \\
Cardiorrespiratório & & 2 & - & 6 \\
Digestório & & - & - & 1 \\
Genitourinário & 3 & - & - \\
NDN & - & - & 1 \\
\hline
\end{tabular}

$\mathrm{NDN}=$ nada digno de nota.

Quanto ao exame ultrassonográfico, foram realizados no total 11 exames, resultando em 13 diagnósticos, sendo 11/13 (85\%) em mamíferos e 02/13 (15\%) em répteis, distribuídos de acordo com o sistema acometido apresentado na tabela abaixo (Tab. 4)

Tabela 4. Número de exames ultrassonográficos realizados dentro de cada classe por sistemas

\begin{tabular}{lcc}
\multicolumn{1}{c}{ Sistema/ Classe } & Répteis & Mamíferos \\
\hline Digestório & 1 & 3 \\
Tegumento comum & - & 2 \\
Genitourinário & - & 5 \\
NDN & 1 & 1 \\
\hline
\end{tabular}

$\mathrm{NDN}=$ nada digno de nota.
Ao se analisarem as alterações observadas no exame radiográfico em cada sistema para cada classe, as alterações mais encontradas em mamíferos foram as fraturas de tíbia (11/ 74) e fêmur (9/74). Nas aves, foram as fraturas de tibiotarso $(17 / 83)$, rádio $(10 / 83)$ e úmero $(9 / 83)$. Já nos répteis, a maior incidência foi de pneumonia $(5 / 34)$ e fratura de fêmur (5/34), seguidas por fraturas de tíbia (4/34) e animais com corpo estranho no trato gastrointestinal (4/34), conforme pode ser observado na Tab. 5 . 


\section{Bortolini et al.}

Tabela 5. Número de exames radiográficos distribuídos de acordo com a classe, o sistema acometido e as alterações encontradas

\begin{tabular}{|c|c|c|c|c|}
\hline Sistema & Patologia & Aves & Mamíferos & Répteis \\
\hline \multirow{3}{*}{ Digestório } & Corpo estranho & 8 & 1 & 4 \\
\hline & Má oclusão & - & 1 & - \\
\hline & Fecaloma & - & 1 & - \\
\hline \multirow{5}{*}{ Genitourinário } & Distocia & - & 1 & 2 \\
\hline & Gestação & - & 2 & - \\
\hline & Cálculo vesical & - & 1 & - \\
\hline & Cálculo renal & - & 1 & - \\
\hline & Cistite & - & 1 & - \\
\hline \multirow{6}{*}{ Cardiorrespiratório } & Pneumonia & 5 & - & 5 \\
\hline & Broncopneumonia & 1 & 2 & 1 \\
\hline & Opacificação campos pulmonares & 1 & - & 1 \\
\hline & Calcificação saco aéreo & 1 & - & - \\
\hline & Aerosaculite & 3 & - & - \\
\hline & Cardiopatia & 1 & - & - \\
\hline \multirow{7}{*}{ Neurológico } & Luxação cervical & - & - & 2 \\
\hline & Subluxação coluna lombar & - & - & 1 \\
\hline & Trauma craniano & 2 & 2 & - \\
\hline & Fratura vertebral & - & 3 & - \\
\hline & Doença do disco intervertebral & - & 3 & - \\
\hline & Espondilose deformante & - & 1 & - \\
\hline & Traumático & & & \\
\hline & Fratura & & & \\
\hline & Clavícula & - & 1 & - \\
\hline & Escápula & - & 1 & - \\
\hline & Úmero & 9 & 3 & 5 \\
\hline & Tíbia/tíbiotarso & 17 & 11 & 4 \\
\hline & Fíbula & 2 & 2 & 2 \\
\hline & Fêmur & 1 & 9 & 2 \\
\hline & Pelve & - & 2 & - \\
\hline & Cauda & - & - & 1 \\
\hline & Rádio & 10 & 6 & - \\
\hline & Ulna & 8 & 3 & - \\
\hline & Tarso & & 2 & \\
\hline & Metatarso & 1 & 2 & - \\
\hline & Metacarpo & 1 & 1 & - \\
\hline Osteomuscular & Luxação & & & \\
\hline & Interfalangeana & - & 1 & 1 \\
\hline & Metacarpofalangeana & - & 1 & - \\
\hline & Sacroilíaca & 1 & 1 & - \\
\hline & Coxofemoral & - & 2 & - \\
\hline & Escapuloumeral & 1 & & - \\
\hline & Umerorradioulnar & 1 & & - \\
\hline & Tibiotarsometatarso & 1 & 1 & - \\
\hline & Tibiotársica & - & 1 & - \\
\hline & Radiocárpica & - & 1 & - \\
\hline & Metabólico & & & \\
\hline & Osteopenia & 1 & 2 & 2 \\
\hline & Deformidade de coluna & - & - & 1 \\
\hline & Infeccioso & & & \\
\hline & Artrite & 1 & - & - \\
\hline & Osteomielite & 1 & 2 & - \\
\hline & Lesão de tecidos moles & 2 & - & - \\
\hline & Fratura bico & 1 & - & - \\
\hline Iegumento comum & Neoplasia cutânea & 1 & - & - \\
\hline & Lise de bico & 1 & - & - \\
\hline
\end{tabular}


A quantidade de requisições de exames imaginológicos em animais selvagens por parte dos especialistas da área tem aumentado significativamente. $\mathrm{O}$ principal fator limitante no diagnóstico das enfermidades é a inexistência ou a escassez de descrições de estudos anatômicos dos diferentes grupos de animais selvagens, devido à grande variação anatômica entre eles.

De acordo com Pinto (2007), mesmo quando se agrupam os animais por similaridade dentro das classes (aves, répteis e mamíferos), persiste ainda uma grande variação anatômica entre os membros de cada grupo. O conhecimento dos padrões de normalidade é essencial para o reconhecimento das alterações que podem estar presentes nas diferentes doenças. Dessa forma, para direcionar o diagnóstico imaginológico dos animais selvagens, muitas vezes são imprescindíveis as comparações entre as imagens da anatomia normal registradas em livros, atlas ou artigos.

Atualmente, a maioria dos exames de animais selvagens solicitados ao Setor de Diagnóstico por Imagem da Unesp - Campus Botucatu são os radiográficos. Neste estudo, foi observado que cerca de $90 \%$ dos exames tomográficos e $80 \%$ dos ultrassonográficos foram realizados com o intuito de pesquisa científica, o que demonstra o aumento no interesse para padronização anatômica desses indivíduos. Entre os exames provenientes da rotina, os mais solicitados foram os radiográficos e os tomográficos para avaliação de lesões pós-traumáticas que acometeram os sistemas osteomuscular e neurológico.
O exame ultrassonográfico foi a modalidade menos requisitada, principalmente em répteis e aves. Segundo Augusto (2007), a avaliação ultrassonográfica pode ser dificultada por particularidades anatômicas, como penas, escamas, cascos, sacos aéreos, espessura da pele, depósitos de gordura e o próprio porte do animal. Essas particularidades podem interferir no contato do transdutor com a pele, dificultando ou impossibilitando a passagem das ondas sonoras ou o retorno dos ecos, o que impede a formação da imagem.

A maior utilização dos meios de diagnóstico por imagem em animais selvagens é para avaliação de traumas, como foi demonstrado neste estudo. Embora os exames radiográficos sejam mais comumente utilizados na rotina, ainda são necessários estudos em diversas espécies para garantir uma qualidade diagnóstica satisfatória para esse exame.

Com o avanço das pesquisas visando à descrição anatômica por meio de novas ferramentas imaginológicas, como a ultrassonografia, a tomografia computadorizada e a ressonância magnética, será possível uma utilização maior dessas modalidades para o diagnóstico de alterações relacionadas aos diversos sistemas.

Palavras-chave: ultrassonografia, radiologia, tomografia computadorizada, animais silvestres, diagnóstico por imagem

\begin{abstract}
The aim of this study was to retrospectively evaluate in the casuistry on class and systems frequently diagnosed in wild animals that were sent to the FMVZ-UNESP-Botucatu diagnostic imaging service. The class of birds was the most referred to the centers, followed by the mammals and reptiles. The majority of the requested tests were the radiographs and in a minor scale the tomography and ultrasound. Although the birds were the greatest number of animals sent to the service, mammals were the most radiographed for wound control. The most frequently observed system was the musculoskeletal caused by trauma, especially in birds. The radiograph was the most exam initially indicated, then the animal could be forwarded to other imaging modalities, which in wild animals medicine is still limited to research.
\end{abstract}

Keywords: ultrasonography, radiology, computed tomography, wild animals, diagnostic imaging 


\section{REFERÊNCIAS}

AUGUSTO, A.Q. Ultra-sonografia. In: CUBAS, Z.S.; SILVA, J.C.R.; CATÃO-DIAS, J.L. (Eds), Tratado de animais selvagens - Medicina Veterinária. São Paulo: Roca. 2007. p.879-895.

GUMPENBERGER, M.; HENNINGER, W. The use of computed tomography in avian and reptile medicine. Semin. Avian Exotic pet., v.10, p.174180, 2001.

MACKEY, E.B.; HERNANDEZ-DIVERS, S.J.; HOLLAND, M.; FRANK, P. Clinical Technique: Application of computed tomography in zoological medicine. J. Exotic Pet Med., v.17, p.198-209, 2008.

MITCHELL, M. Diagnostic imaging considerations for reptiles, 2009. Disponível em: $<$ http://veterinarycalendar.dvm360.com/avhc/con tent/printContentPopup.jsp?id=607407>.

Acessado em: 07 jul. 2011.
PINTO, A.C.B.C.F. Radiologia. In: CUBAS, Z.S.; SILVA, J.C.R.; CATÃO-DIAS, J.L. Tratado de animais selvagens - Medicina Veterinária. São Paulo: Roca. 2007. p.896-919.

SAINSBURY, A.W.; GILLI, C. Ultrasonography anatomy and scanning technique of the coelomic organs of the bosc monitor (varanus exanthematicus). J. Zoo Wildlife Med., v.22, p.421-433, 1991.

STETTER, M.D. Ultrasonography. In: MADER, D.R. (Eds) Reptile medicine and surgery. W.B. Philadelphia: Saunders, 2006. p.665-674.

SUEDMEYER, K. Diagnostic imaging of exotic animals, 2006. Disponível em: <http://www.searchpdf.info/ebooks/anatomy-indiagnostic-imaging.html >. Acessado em: 29 jun. 2011.

VALENTE, A.L.S. Diagnostic imaging of the Loggerhead sea turtle, Caretta caretta. 2007. 139f. PhD Thesis. Universitat autônoma de Barcelona, Spain. 